\title{
Análise critica comparativa dos achados ultra-sonográificos, vídeo histeroscópicos e histológicos no diagnóstico das patologias endometriais
}

Autor: Nilton José Leite

Orientador: Prof. Dr. Rogério Dias

Dissertação de Mestrado apresentada ao Curso de Pós-Graduação do Departamento de Ginecologia e Obstetrícia da Faculdade de Medicina de Botucatu - UNESP em 3 de dezembro de 1999.

Objetivos: Realizar uma análise crítica comparativa da precisão diagnóstica da ultra-sonografia, histeroscopia e biópsia na avaliação da cavidade endometrial, dentro da nossa realidade, nas pacientes atendidas no Serviço de Ginecologia do Hospital das Clínicas da Faculdade de Medicina de Botucatu - UNESP, no período entre os anos de 1995 a 1999.

Métodos: Foi realizado estudo retrospectivo de cento e oitenta e quatro (184) pacientes com suspeita de alterações endometriais que foram submetidas concomitantemente à ultra-sonografia transvaginal, histeroscopia e biópsia com cureta de Novak. Em sessenta e quatro (64) casos, foi realizada a exérese da lesão, por ressecção video-histeroscópica ou histerectomia. Para verificar a concordância entre os métodos, foi utilizado a estatística Kappa. Para comparar as proporções de alterações obtidas nos métodos, foi utilizada a estatística $\chi^{2}$ de Mc Nemar, confrontando-se os métodos dois a dois.

Resultados: A ultra-sonografia teve sensibilidade de $76,77 \%$, especificidade $43,53 \%$, valor preditivo positivo de $38,71 \%$ e valor preditivo negativo de $38,33 \%$. Para a histeroscopia encontramos sensibilidade de $79,80 \%$, especificidade de $32,94 \%$, valores preditivos positivo de 41,91\% e negativo de 38,33\%. Quanto à biópsia encontramos sensibilidade, especificidade, valores preditivos positivos e negativos respectivamente de $30,15 \%, 100,00 \%, 0,00 \%$ e $86,27 \%$. Os casos de carcinoma endometrial foram detectados por todos os métodos.

Conclusão: A histeroscopia e a ultra-sonografia apresentaram precisão diagnóstica semelhantes, porém do ponto de vista prático, a histeroscopia foi melhor para diferenciar lesões como pólipos, miomas, hiperplasias focais e neoplasia endometrial. Não houve nenhuma falha diagnóstica da ultra-sonografia quando o eco endometrial foi menor ou igual a $5 \mathrm{~mm}$. A biópsia mostrou-se insuficiente para o diagnóstico de pólipos, miomas submucosos, hiperplasias focais e endométrio atrófico. Nos casos de atrofia endometrial, a histeroscopia mostrou-se de grande importância para tranqüilizar o médico e a paciente.

Palavras-chave: Endométrio. Ultra-sonografia transvaginal. Histeroscopia. Sangramento uterino anormal. Sangramento pós-menopausa. Hiperplasia de endométrio.

\section{Análise comparativa dos aspectos clínicos, laparoscópicos e histopatológicos da endometriose} pélvica.

Autor: André Luís Fontana

Orientador: Prof. Dr. Rogério Dias

Tese apresentada ao Curso de Pós Graduação em Ginecologia e Obstetrícia - Departamento de Ginecologia e Obstetrícia - Faculdade de Medicina de Botucatu - UNESP em 11 de fevereiro de 2000.

Objetivos: Comparar os aspectos clinicos, achados laparoscópicos e histopatológicos em pacientes com endometriose pélvica atendidas no Setor de Endoscopia Ginecológica e Planejamento Familiar da Faculdade de Medicina de Botucatu-UNESP, no periodo entre os anos de 1994 à 1998.

Métodos: Cento e dez (110) pacientes submetidas à videolaparoscopia por algia pélvica, dispareunia e dismenorréia $(n=63)$, infertilidade $(n=39)$ e massa anexial $(\mathrm{n}=8)$ foram selecionadas retrospectivamente, por apresentarem alterações peritoneais e ovarianas sugestivas de endometriose pélvica. Os dados clínicos que pudessem levar à suspeita clínica de endometriose foram minuciosamente obtidos dos prontuários médicos dos arquivos da Faculdade. Um total de 244 biópsias obtidas das diferentes lesões peritoneais encontradas, foram revistas criteriosamente pelo Departamento de Patologia da Faculdade de Medicina de Botucatu. Os resultados positivos foram analisados estatisticamente através dos métodos $\mathrm{P}$ (probalidade) e $\chi^{2}$ de Mc Nemar e, procurou-se dar ênfase à incidência, ao quadro clínico, à macroscopia, ao grau das lesões e à anatomia patológica.

Resultados: A maioria das pacientes apresentava sinais e sintomas clínicos sugestivos da doença sendo a algia pélvica o sintoma mais freqüente. A faixa etária onde houve a maior probabilidade de ocorrer lesões típicas foi de 25 a 35 anos; e atípicas acima de 35 anos. A incidência das lesões foi maior na raça branca $(80,9 \%)$. Observou-se também tendência à associação 